\title{
SCIDiC
}

International Journal of Dentistry and Oral Science (IJDOS)

ISSN: 2377-8075

\section{Preference Of Parents Towards Type Of Topical Fluoride Application For Children With Primary Dentition}

Research Article

\author{
Navya Khanna ${ }^{1}$, Vignesh Ravindran ${ }^{2 *}$
}

${ }^{1}$ Saveetha Dental College and Hospitals, Saveetha Institute of Medical and Technical Sciences (SIMATS), Saveetha University, Chennai- 77, India.

${ }^{2}$ Senior Lecturer, Department of Pediatric and Preventive Dentistry, Saveetha Dental College and Hospitals, Saveetha Institute of Medical and Technical Sciences [SIMATS], Saveetha University, Chennai- 77, India.

\section{Abstract}

Introduction: Dental caries is the most prevalent oral hygiene concern in children. Fluoride application has played a major role in preventing caries by identifying the high risk population and use of fluoride on them has controlled caries to a considerable extent. Fluoride is used as a chemotherapeutic agent for caries prevention. Fluoride varnish and gel are types of topical fluoride application.

Aim: To assess the acceptance of parents to fluoride treatment for their children with primary teeth.

Materials And Methods: Study conducted in a university setting with the sample size of 522. He study was performed as a retrospective study under a university setting in the outpatient department of Pediatric and Preventive Dentistry. Data was collected by reviewing patient records and analysed data of 56000 patients between June 2020 to Feb 2021. Verification of the data was done with the presence of additional reviewers. Descriptive statistics and comparison with gender of patient using chi-square test was done in SPSS software.

Results And Discussion: Out of 522 patients, 153 of them were aged five years. 52.3\% of them were males and $47.7 \%$ of females, thus showing a male predilection. A majority of $79.1 \%$ of the parents preferred fluoride varnish as it is more preferred for preschool kids. There was no difference in preference of topical fluoride application based on gender although this result was found to be statistically significant $(\mathrm{p}<0.05)$.

Conclusion: Within the limitations of the present study, Fluoride varnish was more commonly preferred when compared to fluoride gel in children with primary dentition. There was no difference in the type of fluoride application based on gender of the child.

Keywords: Primary Teeth; Fluoride; Primary Dentition.

\section{Introduction}

Dental caries is an oral health problem caused by plaque buildup on the tooth surface, which converts sugar from food and beverages into acid, causing tooth damage. It is considered a major health problem affecting 2.4 billion people worldwide in the year 2020 [1]. It is linked to poor oral hygiene, diet and infrequent dental visits [2]. it is a widespread health problem in children [3], prevailing around 60 to $90 \%$ [4]. Moreover, dental caries influences a child's quality of life psychologically [5] exposing him or her to pain and discomfort at an early age. Children with caries were found to be 1.3 times more likely to experience oral health related impact on the quality of their lives [6].

The use of fluoride in various methods such as water fluoridation, toothpaste, sealants, mouth rinses, professional topical agents is considered a measure of great importance for the prevention of dental caries, owing to its anti-cariogenic properties [7]. Fluoride use is currently recognised as the main factor responsible for significant decline of caries prevalence that has been observed in the last few decades. Fluoride therapy has been the cornerstone of caries preventive strategies since the introduction of water fluoridation scheme [8]. Fluoride plays a role in the development and growth of carries, including initiation and progression. Intensive

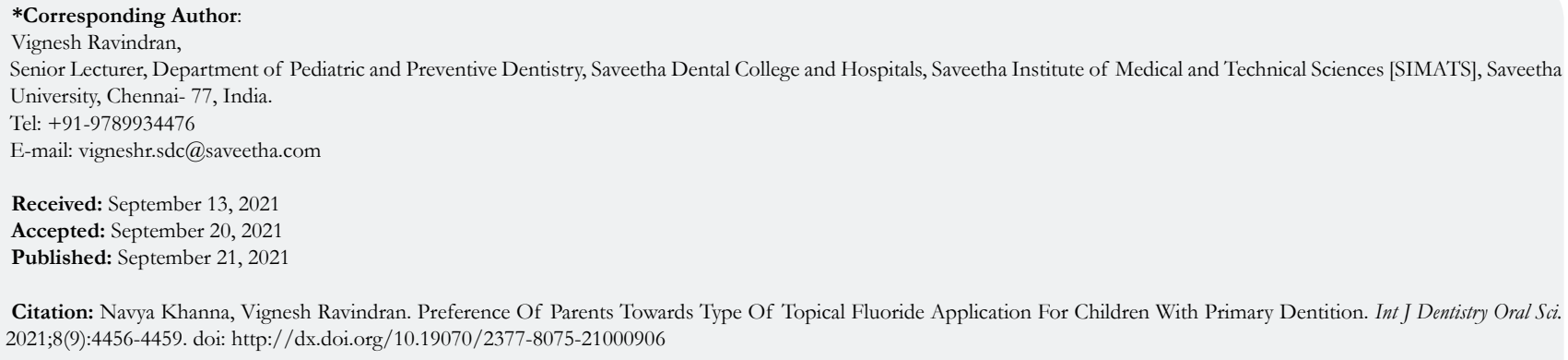

Copyright: Vignesh Ravindran 2021 . This is an open-access article distributed under the terms of the Creative Commons Attribution License, which permits unrestricted use, distribution and reproduction in any medium, provided the original author and source are credited. 
laboratory and epidemiological research on the mechanism of action of fluoride in preventing caries indicates fluoride's predominant effect is topical, which occurs mainly through promotion of remineralisation of early carious lesions and by reducing sound tooth enamel demineralisation [9]. Fluoride is now used in a variety of ways, each with its own prescribed concentration, duration, and dose schedule.

Fluoride varnish has been described as the most convenient form of professional use of topical fluoride in preschool children based on the premise that they are easy to apply and well tolerated [10]. The application time of varnish varies from 1 to 4 minutes. Varnish acts by hardening on contact with saliva and thereby forcing the film that sticks to the dental surface. As a result, it will stay on the enamel surface for several hours [11]. It is easy to apply and well tolerated by children [12]. Fluoride gel is another formulation of fluoride widely used in young adults [13]. The gel is usually placed on a tray of foam material, which the child or young adult has to keep in their mouth and bite it for four minutes [14]. Due to the risk of toxicity, fluoride gel treatment is not given to children below six years of age [15].

In addition to its established cariostatic effect, fluoride is a hazardous substance when excessive intake occurs by ingestion of a large dose. It provokes effects ranging from nausea, vomiting, abdominal pain to even death [16]. Lower doses taken chronically can affect the developing mineralised dental tissues, thus resulting in dental fluorosis, and hypomineralization of enamel clinically, characterised by opacity, pitting or staining. A potential risk for fluorosis may be the inappropriate use of fluoride toothpaste or mouth rinses in young children who may not be able to expectorate it adequately [17]. Therefore, parent or knowledge with respect to dental visits of the child, choice of toothpaste, quantity, and requisite are vital.

The aim of this research is to look at the percentage of parents who accept fluoride treatment for their children, as well as the gender differences and options available to them, in children with primary teeth.

\section{Materials and Methods}

This study was a retrospective study conducted in a University setting under the outpatient department of Pediatric and Preventive Dentistry. The ethical approval was obtained from the Institution Ethical Review Board Approval. Informed consent was obtained from the parents or guardian regarding usage of the clinical data for research purposes.

The required data of patients procured by reviving patient records from June 2019 to February 2021 and reviewed. These patients were from the out-patient department of Paediatric Dentistry. The exclusion criteria were children under specific care, incomplete data and incomplete photographic evidence. Inclusion criteria were children with primary dentition only who underwent topical fluoride application therapy.

Digital entry of clinical examination, intraoral photographs of the oral cavity and the treatment procedure were assessed. The data collected (digital entry and intraoral photographs) was verified by an external additional reviewer. Sampling bias was minimised by doing simple random sampling. There was no resolution of conflict. The censored data was discarded. If any error in data entry or patient details or clinical data were noticed, that case sheet was excluded from the study.

The tabulated data from the Excel is important to SPSS for statistical analysis. The software used was SPSS version 22, IBM software, Chicago. Descriptive statistics and correlation statistics using chi-square test were tabulated and graphically represented.

\section{Results \& Discussion}

The total number of paediatric patients in the study was 522, since only children with primary dentition are involved. Among them, 413 individuals were subjected to fluoride varnish and 109 to fluoride gel. The most common age for treatment was 4 to 5 years. Among all patients, 273 were males and 249 female.

The mean age of children was 4.3 years. Among the patients reported $52.3 \%$ were males and $47.7 \%$ of females (Figure 1). From analysing the preference of fluoride treatment obtained $79.1 \%$ of the parents preferred varnish while $20.9 \%$ preferred gel (Figure 2). On comparison of the preference of fluoride application with gender of the patient, no statistical difference was found $(p>0.05)$ with more parents preferring fluoride varnish for both male and female children. (Figure 3).

Topical application of fluoride gel has been used widely as a measure for the intervention of caries in dental clinics and school based programs for over three decades. Dental preventive therapy should start early in a child's life. The needful early intervention is to reduce the oral diseases and mandate the involvement of parents in the preventive process [18]. Studies have reported low parental knowledge and a poor attitude towards oral health are associated with an experience of high caries in young children [19, 20]. It is a requirement that parents have a positive perspective towards dentistry that it will have a good impact on the child's oral health.

From the results, it was reported that the mean age of the study for children undergoing topical fluoride treatment was five years.

Figure 1. The bar graph represents the gender distribution of the patients. The orange represents the percentage of females $(47.7 \%)$ in the population while the yellow represents the percentage of males $(52.3 \%)$.

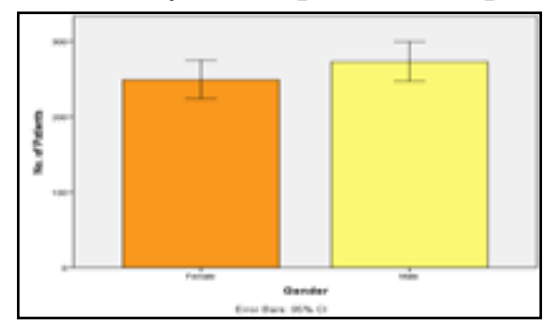


Figure 2. The bar graph represents the choice of preference of fluoride varnish and fluoride gel. The blue represents the percentage of parents who preferred fluoride gel $(20.9 \%)$ in the population while the green represents the percentage of parents who preferred fluoride varnish $\mathbf{( 7 9 . 1 \% )}$.

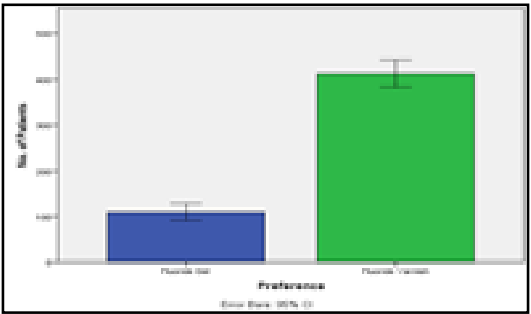

Figure 3. The bar graph represents the correlation between the gender and preference of type of topical fluoride application. The $\mathrm{X}$-axis represents the gender while the $\mathrm{Y}$-axis represents the number of patients. There was no difference in preference of topical fluoride application based on gender although this result was found to be statistically significant.

(Pearson's chi square value: 8.212, df:1, p-value: $0.003(\mathrm{p}<0.05)$. Hence, statistically significant.)

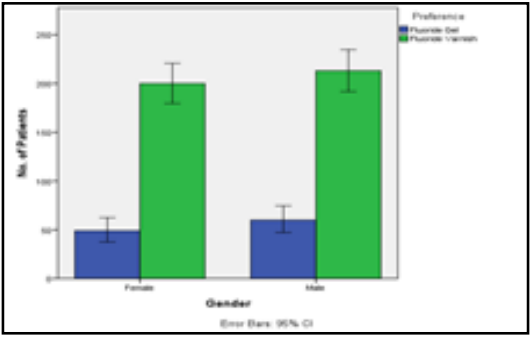

This was in keeping with the study by Ramya et al., [16]. It is due to fluoride being prescribed for children from ages 5 to 17 years. Fluoride varnishes are recommended for pre-school going children. Since a large population was children with primary dentition that is kids below the age of six years, the prevalence of vanishes was more.

The gender distribution study reveals the mail predilection in the acceptance of right treatment. This can be attributed to the fact that the studies performed Unicentre, hence the unequal distribution of population. No previous literature was observed to have interpreted similar findings.

Fluoride varnishes are easy to use and tolerate, making them one of the most practical ways to avoid and treat fluorosis in preschoolers. The time it takes to apply the varnish ranges from 1 to 4 minutes per user, depending on the amount of teeth present, and the child may close it soon afterward since the varnish hardens as it comes into contact with saliva and forms a coating that adheres to the tooth surface. Patients should, however, refrain from feeding for several hours after the varnish has been applied and not clean their teeth the same day. This causes the varnish to last on the dental enamel for many hours.

The study setting had certain advantages like flexibility in data collection, less expenditure and maximum internal validity. However, the study also has certain limitations, that is unicentric with reduced sample size and minimum external validity.

\section{Conclusion}

Within the limitations of the present study, Fluoride varnish was more commonly preferred when compared to fluoride gel in children with primary dentition. There was no difference in the type of fluoride application based on gender of the child.

\section{Acknowledgement}

The authors are thankful to the Department of Pediatric Dentistry, Saveetha Dental College, Saveetha Institute of Medical and Technical sciences, Saveetha University for providing a platform in expressing their knowledge.

\section{Source of Funding}

The present project was funded by

- Saveetha Dental College, Saveetha Institute of Medical and Technical Sciences, Saveetha University and

- Deepak Bearing Industries, New Delhi

\section{References}

[1]. Mulu W, Demilie T, Yimer M, Meshesha K, Abera B. Dental caries and associated factors among primary school children in Bahir Dar city: a crosssectional study. BMC Res Notes. 2014 Dec 23;7:949.Pubmed PMID: 25540044.

[2]. Fontana M, Young DA, Wolff MS. Evidence-based caries, risk assessment, and treatment. Dent. Clin. N. Am. 2009 Jan 1;53(1):149-61.

[3]. Nota A, Caruso S, Cantile T, Gatto R, Ingenito A, Tecco S, et al. Socioeconomic Factors and Oral Health-Related Behaviours Associated with Dental Caries in Preschool Children from Central Italy (Province of Ascoli Piceno). Biomed Res Int. 2019 Dec 23;2019:7981687.Pubmed PMID: 31930137.

[4]. Tagelsir A, Khogli AE, Nurelhuda NM. Oral health of visually impaired schoolchildren in Khartoum State, Sudan. BMC Oral Health. 2013 Jul 17;13:33.Pubmed PMID: 23866155.

[5]. Marinho VCC, Worthington HV, Walsh T, Clarkson JE. Fluoride varnishes for preventing dental caries in children and adolescents. Cochrane Database Syst Rev. 2013 Jul 11;(7):CD002279.

[6]. Murray JJ, Naylor MN. Fluorides and dental caries. The prevention of oral disease. 1996;3:32-67.

[7]. Weintraub JA, Ramos-Gomez F, Jue B, Shain S, Hoover CI, Featherstone JD, et al. Fluoride varnish efficacy in preventing early childhood caries. J Dent Res. 2006 Feb;85(2):172-6.

[8]. Mishra P, Fareed N, Battur H, Khanagar S, Bhat MA, Palaniswamy J. Role of fluoride varnish in preventing early childhood caries: A systematic review. Dent Res J. 2017 May-Jun;14(3):169-176.Pubmed PMID: 28702057.

[9]. Browne D, Whelton H, O'Mullane D. Fluoride metabolism and fluorosis. J 
Dent. 2005 Mar 1;33(3):177-86.

[10]. Bakar NA, Mamat Z. Parental Knowledge and Practices on Preschool Children Oral Healthcare in Nibong Tebal Penang, Malaysia. JOJ Nursing \& Health Care. 2018;7(4):707-11.

[11]. Al Halabi M. Current Guidelines for the Use of Fluoride in Pediatric Dentistry, A Review. Appl. Clin. Res. Clin. Trials Regul. Aff. 2014 Dec 1;1(3):135-44.

[12]. Ismail AI, Hasson H. Fluoride supplements, dental caries and fluorosis: a systematic review. J Am Dent Assoc. 2008 Nov 1;139(11):1457-68.

[13]. Ramakrishnan M, Shukri M. Fluoride, Fluoridated Toothpaste Efficacy And Its Safety In Children-Review. Int. J. Pharm. Res. 2018 Oct 1;10(04):10914.

[14]. Clark DC. Appropriate uses of fluorides for children: guidelines from the Canadian Workshop on the Evaluation of Current Recommendations Concerning Fluorides. CMAJ. 1993 Dec 15;149(12):1787-93.Pubmed PMID: 8261348.

[15]. Chi DL. Caregivers who refuse preventive care for their children: the relationship between immunization and topical fluoride refusal. Am J Public
Health. 2014 Jul;104(7):1327-33.Pubmed PMID: 24832428.

[16]. Ramya G, Ravindran V, Jeevitha M. Prevalence of parents willingness to take up fluoride treatment for their children, the gender differences and common age of acceptance - A retrospective study. IJRPS. 2020 Oct 14;11(SPL3):1365-70.

[17]. Godel J, Canadian Paediatric Society, Community Paediatrics Committee. The use of fluoride in infants and children. Paediatr Child Health. 2002 Oct 1;7(8):569-72.

[18]. Hugar SM, Govani E, Mohandoss S, Gokhale NS, Kukreja P, Hugar SS. Knowledge attitude and perception among the parents of preschool children regarding oral health and early childhood caries (ECC). academia.edu.

[19]. Hamilton ME, Coulby WM. Oral health knowledge and habits of senior elementary school students. J Public Health Dent. 1991 Dec;51(4):212-9.

[20]. Poutanen R, Lahti S, Seppä L, Tolvanen M, Hausen H. Oral health-related knowledge, attitudes, behavior, and family characteristics among Finnish schoolchildren with and without active initial caries lesions. Acta Odontol Scand. 2007 Apr;65(2):87-96.Pubmed PMID: 17453426. 